\title{
NONSTANDARD MEASURE THEORY: AVOIDING PATHOLOGICAL SETS
}

\author{
BY
}

\author{
FRANK WATTENBERG ${ }^{1}$
}

\begin{abstract}
The main results in this paper concern representing Lebesgue measure by nonstandard measures which avoid certain pathological sets. An (external) set $E$ is $S$-thin if $\operatorname{Inf}\{m A \mid A$ standard, $A \supseteq E\}=0$ and $Q$-thin if Inf $\{* m A \mid A$ internal, $A \supseteq E\}=0$. It is shown that any "finite sample which represents Lebesgue measure avoids every $S$-thin set and that given any $Q$-thin set $E$ there is a *finite sample avoiding $E$ which represents Lebesgue measure. In the last part of the paper a particular pathological set $\boldsymbol{x} \subseteq$ $-[0,1]$ is constructed which is important for the study of approximate limits, derivatives etc. It is shown that every "finite sample which represents Lebesgue measure assigns inner measure zero and outer measure one to this set and that Loeb measure does the same. Finally, it is shown that Loeb measure can be extended to a $\sigma$-algebra including $x$ in such a way that $x$ is assigned zero measure.
\end{abstract}

I. Introduction and preliminaries. Nonstandard Analysis provides us with new techniques for representing measures. The first such technique to be developed was that of representing probability measures as *finite counting measures ([1], [3], [7] and [13]) as follows. Given a probability measure $\nu$ one finds a *finite set $F$ such that for every standard measurable set $E, \nu(E)=$ $\mathrm{St}(\|F \cap * E\| /\|F\|)$, where $\|A\|$ denotes the *ardinality of $A$ for any (internal) *finite set $A$. This enables one to use *finite combinatorial techniques to obtain results in probability theory. The *finite sets constructed in these papers are obtained by ad hoc saturation arguments containing a great deal of leeway.

More recently Peter Loeb [4] has applied the Carathéodory Extension process in ${ }^{*} \mathbf{R}$ as another technique for doing Nonstandard Measure Theory. Thus, we have a number of powerful techniques for representing measures via Nonstandard Analysis. This paper is motivated by the problem of representing Lebesgue measure by nonstandard measures which are concentrated on the complements of specific "pathological" sets. This problem arises, for example, in the investigation of problems involving "approximate" properties of functions. In $\$ I V$ we construct a set $\varkappa$ which is important for such problems. The work in this paper was originally motivated by the problem of

Received by the editors October 16, 1977 and, in revised form, May 23, 1978.

AMS (MOS) subject claserifications (1970). Primary 02H25, 26A98, 28A10; Secondary $28 A 75$.

'This work was partially supported by NSF grant No. MCS77-02262. 
representing Lebesgue measure by a nonstandard measure which was concentrated on the complement of $\Re$.

In §II of this paper we give a new construction of *finite sets representing Lebesgue measure. In \$III we show that this construction can be used to construct such *finite sets avoiding any given set of Loeb measure [4] zero. In $\S I V$ we show that the set $\Re$ has inner measure zero and full outer measure with respect to any *finite counting measure representing Lebesgue measure. This same proof shows that $\Re$ has inner Loeb measure zero and full outer Loeb measure. This result is in striking contrast with standard measure theory where every "natural" set is measurable.

In $\S \mathrm{V}$ we show that Loeb measure can be extended to a measure $m_{E}$ defined on a $\sigma$-algebra which includes $\Re$ in such a way that $m_{E}(\tau)=0$. This gives a solution to the problem of representing Lebesgue measure by a nonstandard measure which gives $\Re$ measure zero, but this solution is considerably weaker than one might like.

The author's work on this paper has been helped by conversations with a number of people including Bob Anderson, Lucy Garnett, Don Geman, David Hayes and Joel Zinn.

This paper will presuppose familiarity with one of the basic books on Nonstandard Analysis (e.g. [5], [8], or [12]). $\mathfrak{N}$ will denote a higher order model including the real numbers $R$ and a given measure space $X$. $* \mathfrak{T}$ will denote a $\kappa$-saturated elementary extension of $\mathfrak{N}$ where $\kappa$ is any cardinal greater than that of the universe of $\mathfrak{K}$. If $P$ is an entity in $\mathscr{N}$ the corresponding entity in $* \mathfrak{N}$ will be denoted $* P$. In particular, $F$ will denote the set of finite subsets of $X$ and ${ }^{*} F$ the ${ }^{*}$ finite subsets of ${ }^{*} X$ in $* \Re$, $\omega$ will denote the set $\{0,1,2, \ldots\}$ of natural numbers in $\Re$ and $* \omega$ its extension in $* \Re$. The cardinality function \|\|$: F \rightarrow \omega$ extends to ${ }^{*}\|\|:{ }^{*} F \rightarrow{ }^{*} \omega$ in ${ }^{*} \Re$ where it enjoys the same formal properties as in $\Re$. By abuse of notation we will write $\|A\|$ for $A \in{ }^{*} F$. We will use the usual notation, $\operatorname{St}(t)$, for the standard part of a (nearstandard) point $t \in{ }^{*} R$, and $a \approx b$ for $a$ infinitely close to $b$.

II. Random numbers and Lebesgue samples.

II.1 Definition. A *finite set $F=\left\{x_{1}, x_{2}, \ldots, x_{v}\right\} \subseteq{ }^{*}[0,1]$ is said to be a Lebesgue sample provided for every standard Lebesgue measurable set $E \subseteq$ $[0,1], m E=\operatorname{St}\left(\left\|^{*} E \cap F\right\| / \nu\right)$.

Suppose $(X, m)$ is a (standard) measure space. Throughout this paper $X$ will either be $I=[0,1]$ or the set, $I^{\omega}$, of all sequences in $I$. In the former case we will use Lebesgue measure and in the latter case the Borel measure induced by 


$$
\begin{aligned}
m\left(\left(a_{1}, b_{1}\right) \times\left(a_{2}, b_{2}\right) \times \cdots \times\right. & \left.\times\left(a_{k}, b_{k}\right) \times I \times I \times \ldots\right) \\
& =\left(b_{1}-a_{1}\right)\left(b_{2}-a_{2}\right) \cdots\left(b_{k}-a_{k}\right) .
\end{aligned}
$$

In the spirit of [11] we define a random element of ${ }^{*} X$ as follows.

II.2 Definition. Suppose $(X, m)$ is a measure space. Then $x \in{ }^{*} X$ is said to be $m$-random provided for every standard $m$-null set $A \subseteq X, x \notin^{*} A$.

A straightforward enlargement argument shows, that most points in ${ }^{*} X$ are $m$-random in the following sense.

II.3 THEOREM. There is an internal ${ }^{*} m$-measurable set $S$ such that ${ }^{*} m(S)=$ 0 and every $x \notin S$ is $m$-random.

If $x=\left(x_{1}, x_{2}, \ldots\right)$ is an element of ${ }^{*} I^{\omega}$ and $\nu$ is an infinite integer then $F=\left\{x_{1}, x_{2}, \ldots, x_{v}\right\}$ is a ${ }^{*}$ finite set. The main theorem of this section shows that if $x$ is $m$-random and $\nu$ is large enough $F$ is a Lebesgue sample.

II.4 Theorem. Suppose $x \in{ }^{*} I^{\omega}$ is $m$-random. Then there is an infinite integer $\lambda$ such that for every $\nu \geqslant \lambda, F=\left\{x_{1}, x_{2}, \ldots, x_{\nu}\right\}$ is a Lebesgue sample.

Proof. First, notice that since $x$ is $m$-random, if $i, k$ are distinct positive *integers then $x_{i} \neq x_{k}$. Let $\alpha$ be a fixed positive infinitesimal. Let $E$ be any standard Lebesgue measurable set. By the Strong Law of Large Numbers (see, for example [2]) there is a set $S_{E} \subseteq I^{\omega}$ of measure zero such that $\forall t \notin S_{E}, \operatorname{Lim}_{n \rightarrow \infty}(1 / n) \sum_{i=1}^{n} \chi_{E}\left(x_{i}\right)=m E$. Since $x$ is $m$-random, $x \notin * S_{E}$ so that $\operatorname{Lim}_{n \rightarrow \infty}(1 / n) \sum_{i=1}^{n} \chi_{E}\left(x_{i}\right)=m E$. Thus, there is a positive *integer $\lambda_{E}$ such that $\forall \nu>\lambda_{E}$

$$
\left|\frac{1}{\nu} \sum_{i=1}^{\nu}{ }^{*} \chi_{E}\left(x_{i}\right)-{ }^{*} m E\right|<\alpha .
$$

But $(1 / \nu) \sum_{i=1}^{\nu} \chi_{E}\left(x_{i}\right)=\left\|F \cap \cap^{*} E\right\| / \nu$ where $F=\left\{x_{1}, x_{2}, \ldots, x_{\nu}\right\}$. By a straightforward saturation argument there is a positive *integer $\lambda$ such that for every standard measurable $E \subseteq E, \lambda \geqslant \lambda_{E}$. This $\lambda$ is the desired $\lambda$.

There are a number of other ways to attempt to generate Lebesgue samples. For example, if $\alpha$ is a fixed standard irrational, $\nu$ is an infinite ${ }^{*}$ integer and $x$ is any point in ${ }^{*} I$ the set $F_{\nu}(x)=\{x, x+\alpha, x+2 \alpha, \ldots, x+$ $(\nu-1) \alpha$ ) (where addition in $I$ is modulo one) is a good candidate for a Lebesgue sample. In this case we have the following theorem whose proof is identical to that of Theorem II.4 with the Ergodic Theorem (see, for example, [2]) substituting for the Strong Law of Large Numbers.

II.5 THEOREM. Suppose $\alpha$ is a fixed standard irrational and $x \in{ }^{*} I$, then $x$ is $m$-random if and only if there is a positive *integer $\nu$ such that $F_{\nu}(x)$ is a Lebesgue sample. 


\section{Avoiding thin sets.}

III.1 Definition. Suppose $E$ is a (possibly external) subset of $* I$. $E$ is said to be $S$-thin if for every standard $\varepsilon>0$ there is a standard measurable set $A \subseteq I$ such that $m(A)<\varepsilon$ and $E \subseteq{ }^{*} A . E$ is said to be $Q$-thin if for every standard $\varepsilon>0$ there is an internal *measurable set $A \subseteq{ }^{*} I$ such that ${ }^{*} m(A)$ $<\varepsilon$ and $E \subseteq A$. Notice the union of an (externally) finite number of $S$-thin [resp. $Q$-thin] sets is $S$-thin [resp. $Q$-thin]. A set $E$ is $Q$-thin if and only if ${ }^{0} m(E)=0$ where ${ }^{0} m$ is the Loeb measure obtained from ${ }^{*} m$.

III.2 ExAMPLES. (i) If $F$ is any Lebesgue sample, $F$ is $Q$-thin since being ${ }^{*}$ finite, it is ${ }^{*}$ measurable and ${ }^{*} m(F)=0$. However, $F$ is not $S$-thin since $F \subseteq * A$ implies $m(A)=\operatorname{St}(\|F \cap * A\| /\|F\|)=1$.

(ii) Suppose $A \subseteq I$ is a standard Lebesgue null set. Let $E(A)=\{x \in$ * $I \mid \operatorname{St}(x) \in A\}$. Then $E(A)$ is $S$-thin.

Proof. Given a standard $\varepsilon>0$ there is a standard open set $U$ such that $A \subseteq U$ and $m(U)<\varepsilon$. But $E(A) \subseteq{ }^{*} U$.

(iii) Suppose $A \subseteq I$ is any standard Lebesgue measurable set. Then $E(A) \backslash$ * $A$ is $S$-thin.

Proof. The proof is a straightforward modification of (ii).

(iv) Suppose $A \subseteq I$ is any standard Lebesgue measurable set. Then $E(A) \Delta^{*} A$ is $S$-thin.

Proof. $E(A) \Delta^{*} A=(E(A) \backslash * A) \cup(E(I \backslash A) \backslash *(I \backslash A))$.

If $A$ is a set of "bad" points we would like to find a Lebesgue sample $F$ which "avoids" $A$ in the sense that $\|A \cap F\| /\|F\|$ is infinitesimal. Unfortunately, $A$ is usually external so $\|A \cap F\|$ is undefined. Thus, the best we can do is the following.

III.3 Definition. A Lebesgue sample $F$ is said to avoid a (possibly external) set $E$ provided for every standard $\varepsilon>0$ there is an internal set $B \subseteq F$ such that $\|B\| /\|F\|<\varepsilon$ and $F \cap E \subseteq B$.

III.4 THEOREM. Suppose A is a (possibly external) subset of *I. Then

(i) If $A$ is $S$-thin, every Lebesgue sample avoids $A$.

(ii) If $A$ is $Q$-thin, there exists a Lebesgue sample which avoids $A$.

Proof. (i) The proof of (i) is completely straightforward.

(ii) The proof is a modification of Theorem II.4. Let $\alpha$ be a positive infinitesimal as in that proof.

For each standard Lebesgue measurable set $E$, let $S_{E}$ be as in the proof of Theorem II.4.

Since $A$ is $Q$-thin there are internal *measurable sets $A_{1}, A_{2}, \ldots$ such that ${ }^{*} m A_{n}<1 / n$ and $A \subseteq A_{n}$. Notice, although each $A_{n}$ is internal, the sequence $A_{1}, A_{2}, \ldots$ is external and is indexed by the standard positive integers.

By the *(Strong Law of Large Numbers) there is an internal *measurable 
set $S_{n}$ such that ${ }^{*} m S_{n}=0$ and for every $t \notin S_{n}$,

$$
{ }_{m \rightarrow \infty}^{*} \operatorname{Lim}_{m \rightarrow}\left(\frac{1}{m} \sum_{i=1}^{m} \chi_{A_{n}}\left(t_{i}\right)\right)={ }^{*} m A_{n}<\frac{1}{n} .
$$

By a straightforward saturation argument there is a (Lebesgue null) set $S$ such that

(i) For every $n, S_{n} \subseteq S$.

(ii) For every standard Lebesgue measurable set $E,{ }^{*} S_{E} \subseteq S$.

Now, let $x \in{ }^{*} I^{\omega}$ be any point such that $x \notin S$. For each standard Lebesgue measurable set $E$, let $\lambda_{E}$ be as in the proof of Theorem II.4.

For each $n$, since $x \notin S_{n}$

$$
{ }^{*} \operatorname{Lim} \frac{1}{m}\left(\sum_{i=1}^{m} \chi_{A_{n}}\left(x_{i}\right)\right)=m^{*} A_{n}<\frac{1}{n} .
$$

Hence, there is a $\lambda_{n}$ such that $\forall \nu \geqslant \lambda_{n}$

$$
\frac{1}{\nu} \sum_{i=1}^{\nu} \chi_{A_{n}}\left(x_{i}\right)<\frac{1}{n}
$$

By a straightforward saturation argument there is a positive *integer $\lambda$ such that

(i) For every standard Lebesgue measurable $E, \lambda>\lambda_{E}$.

(ii) For every $n, \lambda>\lambda_{n}$.

Then for any $\nu>\lambda$ the set $F=\left\{x_{1}, x_{2}, \ldots, x_{\nu}\right\}$ is a Lebesgue sample and by (*)

$$
\frac{1}{\nu}|| F \cap A_{n} \mid<\frac{1}{n} .
$$

But since $A \subseteq A_{n}$ this implies that $F$ avoids $A$. This completes the proof.

It is well known [8] that a standard function $f: I \rightarrow R$ is continuous if and only if for every $x \in{ }^{*} I,{ }^{*} f(x) \approx f(\operatorname{St}(x))$. In the present context Littlewood's Second Principle [9]: Every measurable function is nearly continuous; takes the following form.

III.5 Corollary. Suppose $f: I \rightarrow R$ is a standard Lebesgue measurable function and $F$ is any Lebesgue sample. Then $F$ avoids the set, $B=\left\{\left.x\right|^{*} f(x) \approx\right.$ $f(\operatorname{St}(x))\}$.

Proof. By Lusin's Theorem there are standard continuous functions $f_{1}$, $f_{2}, \ldots$ such that $m\left(A_{n}\right)<1 / n$ where $A_{n}=\left\{x \mid f(x) \neq f_{n}(x)\right\}$. For each $n$ choose a standard open set $U_{n}$ such that $A_{n} \subseteq U_{n}$ and $m\left(U_{n}\right)<2 / n$. Suppose $x \in B$. Then since ${ }^{*} f_{n}(x) \approx f_{n}(\operatorname{St}(x))$ either ${ }^{*} f_{n}(x) \neq{ }^{*} f(x)$ in which case $x \in{ }^{*} A_{n}$ or $f_{n}(\operatorname{St}(x)) \neq f(\operatorname{St}(x))$ in which case $\operatorname{St}(x) \in A_{n}$ so that $x \in$ $\mu(\operatorname{St}(x)) \subseteq{ }^{*} U_{n}$. Thus $B \subseteq{ }^{*} U_{n}$ and the corollary follows from Theorem III.4. 
IV. A particular pathological set. In this section we wish to study a particular pathological set of importance in work involving "approximate" properties. We show here, that the techniques developed thus far are not sufficient for dealing with this set. In the next section, however, we exhibit a method for dealing with this set.

We begin by recalling a few classical definitions [10].

IV.1 Definition. Suppose $A \subseteq[0,1]$ and $a \in[0,1], a$ is said to be a point of dispersion for $A$ provided

$$
\operatorname{Lim}_{\varepsilon \rightarrow 0} \frac{\bar{m}(A \cap(a-\varepsilon, a+\varepsilon))}{2 \varepsilon}=0 .
$$

where $\bar{m}$ denotes outer measure. Suppose $f:[0,1] \rightarrow \mathbf{R}$ and $a \in[0,1] . f$ is said to be approximately continuous at $a$ provided for every $\varepsilon>0, a$ is a point of dispersion for the set $\{y \in[0,1]|f(y)-f(a)|>\varepsilon\}$.

Approximate limits, derivatives, etc. are defined in the obvious way. These notions are much more general than their "exact" counterparts. For example [10], a function $f:[0,1] \rightarrow \mathbf{R}$ is measurable if and only if it is approximately continuous almost everywhere. From the point of view of Nonstandard Analysis we can approach these notions as follows.

IV.2 Definition. Suppose $x \in{ }^{*}[0,1] . x$ is said to be negligible provided there is a standard set $A$ such that

(i) $\operatorname{St}(x)$ is a point of dispersion for $A$.

(ii) $x \in{ }^{*} A$.

We may assume the set $A$ is measurable since the following argument shows that if $x$ is a point of dispersion for $A$ then there is an open set $U \supseteq A \backslash\{x\}$ such that $x$ is a point of dispersion for $U$.

Since $x$ is a point of dispersion for $A$ there is a descending sequence $\varepsilon_{1}, \varepsilon_{2}, \ldots$ such that $\forall \varepsilon<\varepsilon_{n}, \bar{m}(A \cap(x-\varepsilon, x+\varepsilon)) / 2 \varepsilon<1 / n$.

Hence for each $k=0,1,2 \ldots, \bar{m}\left(A \cap\left(x-\varepsilon_{n} / 2^{k}, x+\varepsilon_{n} / 2^{k}\right)\right) 2^{k} / \varepsilon_{n}<$ $1 / n$ and by the definition of outer measure there is an open set $U_{n k} \supseteq A \cap[x$ $\left.-\varepsilon_{n} / 2^{k}, x+\varepsilon_{n} / 2^{k}\right]$ such that $m\left(U_{n k}\right) 2^{k} / \varepsilon_{n}<1 / n$. Let

$$
U_{n}=\bigcap_{k}\left[U_{n k} \cup\left(-\infty, x-\varepsilon_{n} / 2^{k}\right) \cup\left(x+\varepsilon_{n} / 2^{k}, \infty\right)\right] .
$$

Notice $A \subseteq U_{n}$ and for every $\varepsilon<\varepsilon_{n}, m(A \cap(x-\varepsilon, x+\varepsilon)) / 2 \varepsilon<2 / n$.

Let $U^{\prime}=\bigcap_{n}\left[U_{n} \cup\left(-\infty, x-\varepsilon_{n}\right) \cup\left(x+\varepsilon_{n}, \infty\right)\right]$.

Let $U=U^{\prime} \backslash\{x\}$. $U$ is the desired set.

Let $\mathscr{N}$ denote the set of negligible points in ${ }^{*}[0,1]$. The proofs of the following propositions are completely straightforward.

IV.3 Proposition. Suppose $A \subseteq[0,1]$ and $x \in[0,1]$. Then $x$ is a point of dispersion for $A$ if and only if $\mu(x) \cap \cap^{*} A \subseteq \Re$. 
Proof. If $x$ is a point of dispersion for $A$ then by Definition IV.2, $\mu(x) \cap^{*} A \subseteq \Re$.

Conversely, suppose $x$ is not a point of dispersion for $A$. Then

$$
\operatorname{Lim}_{\varepsilon \rightarrow 0} \frac{\bar{m}(A \cap(x-\varepsilon, x+\varepsilon))}{2 \varepsilon}
$$

is not zero. Hence, there is a positive infinitesimal $\alpha$ such that

$$
\frac{{ }^{*} \bar{m}\left({ }^{*} A \cap(x-\alpha, x+\alpha)\right)}{2 \alpha}
$$

is not infinitesimal. But now, a straightforward saturation argument produces a nonstandard $y \in(x-\alpha, x+\alpha) \cap \cap^{*} A$ such that if $x$ is a point of dispersion for the standard set $E$ then $y \notin * E$. Hence $y \notin \Re$ and $\mu(x) \cap{ }^{*} A$ $z \Re$.

We remark in passing, that this proposition implies that the monad of a (standard) point $a$ in the density topology [2A] on $\mathbf{R}$ is $(\mu(x) \backslash \Re) \cup\{x\}$.

IV.4 Proposition. Suppose $f:[0,1] \rightarrow \mathbf{R}$ and $x \in[0,1]$. Then $f$ is approximately continuous at $x$ if and only if $\forall t \in \mu(x) \backslash \mathscr{T},{ }^{*} f(t) \approx{ }^{*} f(x)$.

Thus, we see that the approximate behavior of a function $f$ is reflected in its behaviour off $\Re$, and, so if we could find a Lebesgue sample which avoided $\Re$ we would have a powerful tool relating the approximate behavior of a function with its measure theoretic properties. Unfortunately, the following theorem shows that this is impossible except in a much weaker sense than that of $\$$ III.

IV.5 THEOREM. Suppose $F$ is a Lebesgue sample. Then,

(i) If $H$ is an internal subset of $F \cap \Re,\|H\| /\|F\| \approx 0$.

(ii) If $H$ is an internal subset of $F$ containing $F \cap \Re$ then $\|H\| /\|F\| \approx 1$.

Proof. (i) Suppose $H$ is an internal subset of $F \cap \Re$. We must show $\|H\| /\|F\| \approx 0$.

Suppose not. Let $s$ be a standard positive real such that $\|H\| /\|F\|>s$. We define a standard sequence of intervals $\left[a_{0}, b_{0}\right] \supseteq\left[a_{1}, b_{1}\right] \supseteq \ldots$ such that

(i) $b_{n}-a_{n}=1 / 2^{n}$,

(ii) $\left(\left\|H \cap{ }^{*}\left[a_{n}, b_{n}\right]\right\| /\|F\|\right) \cdot 2^{n}>s$

as follows. Let $\left[a_{0}, b_{0}\right]=[0,1]$. Having found $\left[a_{0}, b_{0}\right] \supseteq \cdots \supseteq\left[a_{n}, b_{n}\right]$, let $c_{n}=\left(a_{n}+b_{n}\right) / 2$. Since

$$
\frac{\left\|H \cap *\left[a_{n}, b_{n}\right]\right\|}{\|F\|} \cdot 2^{n}>s .
$$


either

$$
\frac{\left\|H \cap *\left[a_{n}, c_{n}\right]\right\|}{\|F\|} \cdot 2^{n}>\frac{s}{2} \quad \text { or } \quad \frac{\left\|H \cap *\left[c_{n}, a_{n}\right]\right\|}{\|F\|} \cdot 2^{n}>\frac{s}{2} .
$$

In the first case let $\left[a_{n+1}, b_{n+1}\right]=\left[a_{n}, c_{n}\right]$. Otherwise, let $\left[a_{n+1}, b_{n+1}\right]=$ $\left[c_{n}, b_{n}\right]$.

Let $x$ be the unique (standard) point in $\cap\left[a_{n}, b_{n}\right]$. Notice for every standard $\varepsilon>0$

$$
\frac{\|H \cap *(x-\varepsilon, x+\varepsilon)\|}{\|F\| \cdot 2 \varepsilon}>\frac{s}{4} .
$$

Now, suppose $A$ is any standard measurable set such that $x$ is a point of dispersion for $A$ and $\delta$ is any standard positive real. Let $\Phi_{A, \delta}(t)$ be the sentence

$$
|t-x|<\delta \text { and } t \in H-{ }^{*} A .
$$

Claim. For each such $A, \delta$ there is a $t$ such that $\Phi_{A, \delta}(t)$.

Proof. Since $x$ is a point of dispersion for $A$ there is a $\delta^{\prime}<\delta$ s.t.

$$
\frac{m\left(A \cap\left(x-\delta^{\prime}, x+\delta^{\prime}\right)\right)}{2 \delta^{\prime}}<\frac{s}{8} .
$$

But $F$ is a sample so

$$
\frac{\left\|F \cap * A \cap *\left(x-\delta^{\prime}, x+\delta^{\prime}\right)\right\|}{\|F\| \cdot 2 \delta^{\prime}}<\frac{s}{8} .
$$

Thus,

$$
\frac{\left\|H \cap * A \cap *\left(x-\delta^{\prime}, x+\delta^{\prime}\right)\right\|}{\|F\| \cdot 2 \delta^{\prime}}<\frac{s}{8} .
$$

Hence, by (\#) $H \cap{ }^{*}\left(x-\delta^{\prime}, x+\delta^{\prime}\right) \underline{*} A$ and since $\delta^{\prime}<\delta$,

$$
H \cap *(x-\delta, x+\delta) Z^{*} A .
$$

Now, the sentences $\Phi_{A, \delta}(t)$ are finitely satisfiable since if $x$ is a point of dispersion for $A_{1}, \ldots, A_{n}$ then $x$ is a point of dispersion for $A_{1} \cup A_{2}$ $\cup \ldots \cup A_{n}$.

Let $t$ be a point satisfying every $\Phi_{A, \delta}(t)$. Then $t \in \mu(x), t \in H$, and $t \notin \Re$. $\therefore H \& \Re$.

(iii) Suppose $H$ is an internal subset of $F$ containing $F \cap \Re$. We must show $\|H\| /\|F\| \approx 1$.

Suppose not. Let $s$ be a standard real such that $\|H\| /\|F\|<s<1$. Using an argument similar to that in (i) we find a standard sequence of standard intervals $\left[a_{0}, b_{0}\right] \supseteq\left[a_{1}, b_{1}\right] \supseteq\left[a_{2}, b_{2}\right] \supseteq \ldots$. 
(i) $b_{n}-a_{n}=1 / 2^{n}$.

(ii) $\left(\left\|H \cap{ }^{*}\left[a_{n}, b_{n}\right]\right\|\right) /\|F\| \cdot 2^{n}<s$.

Let $x$ be the unique standard point in $\cap\left[a_{n}, b_{n}\right]$.

We will construct a standard sequence of intervals $J_{1}, J_{2}, \ldots$ such that

(i) $x$ is a point of dispersion for $J=\cup_{1}^{\infty} J_{n}$,

(ii) For each $J_{n},{ }^{*} J_{n} \cap F \underline{Z} H$,

(iii) For every standard $\varepsilon>0 \exists N$ such that $\forall n>N, J_{n} \subseteq(x-\varepsilon, x+\varepsilon)$.

Suppose we have such a sequence. Let $T=\left\{\nu \mid J_{\nu} \cap F \notin H\right\}$. T is an internal set containing every standard $n$. Hence, $T$ contains at least one infinite $\nu$. But $J_{\nu} \subseteq \mu(x)$ and $\mu(x) \cap * J \subseteq \Re$ since $x$ is a point of dispersion for ${ }^{*} J$. Hence, since $J_{v} \in H, \mathscr{R} \in H$. This contradiction will complete the proof. In order to construct the sequence $J_{1}, J_{2}, \ldots$ we need the following lemma.

IV.6 Lemma. Let $F, H$ and $x$ be as above. Suppose $\varepsilon, \delta$ are standard positive reals. Then there is an interval $[u, v]$ such that

(i) $[u, v] \subseteq(x-\varepsilon, x+\varepsilon)$,

(ii) ${ }^{*}[u, v] \cap F \underline{Z} H$,

(iii) $x \notin[u, v]$,

(iv) $(v-u) / d(x,[u, v])<\delta$.

Proof. First choose $n$ large enough so that $\left[a_{n}, b_{n}\right] \subseteq(x-\varepsilon, x+\varepsilon)$. By the construction of $\left[a_{n}, b_{n}\right]$

$$
\frac{\left\|H \cap{ }^{*}\left[a_{n}, b_{n}\right]\right\|}{\|F\|}<s\left(b_{n}-a_{n}\right) .
$$

Since $F$ is a Lebesgue sample

$$
\frac{\left\|F \cap *\left[a_{n}, b_{n}\right]\right\|}{\|F\|} \approx\left(b_{n}-a_{n}\right) .
$$

Let $t$ be the point in $(F \backslash H) \cap\left[a_{n}, b_{n}\right]$ which is farthest from $x$. By (1) and (2) $|t-x|$ is not infinitesimal. Hence, one can easily find a standard interval $[u, v]$ such that $t \in{ }^{*}[u, v]$ and $[u, v]$ satisfies (i)-(iv).

Now, we define the sequence $J_{1}, J_{2}, \ldots$ inductively.

By Lemma IV.6 there is a $J_{1}=\left[u_{1}, v_{1}\right]$ such that

(i) ${ }^{*} J_{1} \cap F \underline{Z} H$,

(ii) $\left(v_{1}-u_{1}\right) / d\left(x, J_{1}\right)<\frac{1}{4}$,

(iii) $d\left(x, J_{1}\right)<\frac{1}{4}$.

We continue inductively using Lemma IV.6 to obtain intervals $J_{n}=\left[u_{n}, v_{n}\right]$ such that

(i) * $J_{n} \cap F \& H$,

(ii) $\left(v_{n}-u_{n}\right)<\left(v_{n-1}-u_{n-1}\right) / 2$, 
(iii) $\left(v_{n}-u_{n}\right) / d\left(x, J_{n}\right)<1 / 2^{n+1}$,

(iv) $0<d\left(x, J_{n}\right)<1 / 2^{n+1}$.

All we need show is that $x$ is a point of dispersion for $J=U J_{n}$.

Given $\varepsilon>0$ choose $N$ large enough so that $1 / 2^{N}<\varepsilon$. Let $\delta=$ $\operatorname{Min}\left(d\left(x, J_{1}\right), d\left(x, J_{2}\right), \ldots, d\left(x, J_{N}\right)\right)$. Suppose $t<\delta$. Let $k$ be the least integer such that $J_{k} \cap(x-t, x+t) \neq \varnothing$. Notice $k>N$.

$$
\begin{aligned}
(x-t, x+t) \cap J & \subseteq \bigcup_{i=k}^{\infty} J_{i}, \\
m((x-t, x+t) \cap J) & <\sum_{i=k}^{\infty}\left(v_{i}-u_{i}\right)<2\left(v_{k}-u_{k}\right) .
\end{aligned}
$$

Since $J_{k} \cap(x-t, x+t) \neq \varnothing, t>d\left(x, J_{k}\right)$

$$
\frac{\left(v_{k}-u_{k}\right)}{t}<\frac{\left(v_{k}-u_{k}\right)}{d\left(x, J_{k}\right)}<\frac{1}{2^{k+1}} \text {. }
$$

Thus

$$
\frac{m((x-t, x+t) \cap J)}{2 t}<\frac{2\left(v_{k}-u_{k}\right)}{2 t}<\frac{1}{2^{k+1}}<\frac{1}{2^{N}}<\varepsilon .
$$

This completes the proof.

Finally, we remark that this proof can be modified to show that $\Re$ has "outer" Loeb measure 1 and "inner" Loeb measure 0 and, hence, is not Loeb measurable.

V. Extensions of Loeb measure. The following construction allows one to extend Loeb measure to a larger $\sigma$-algebra including the set $\Re$ in such a way that $\Re$ is assigned zero measure.

V.1 Definition. Let $\mathcal{E}$ denote the $\sigma$-algebra of 0 -measurable subsets of ${ }^{*}[0,1]$. Suppose $E$ is a subset of ${ }^{*}[0,1]$ of full outer ${ }^{0} m$-measure. (I.e. $\operatorname{Inf}_{E \subseteq A \in \mathfrak{R}}{ } m(A)=1$.)

Let $\mathcal{L}_{E}=\left\{A \subseteq{ }^{*}[0,1] \mid \exists B \in \mathcal{L}, A \cap E=B \cap E\right\}$.

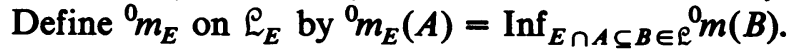

V.2 TheOREM. (i) ${ }^{0} m_{E}(A)={ }^{0} m(B)$ if $B \in \mathcal{L}$ and $A \cap E=B \cap E$.

(ii) For each $A \in \mathcal{L},{ }^{0} m_{E}(A)={ }^{0} m(A)$.

(iii) ${ }^{0} m_{E}$ is a $\sigma$-additive measure on $\mathfrak{L}_{E}$.

(iv) $\mathfrak{L}_{E}$ is complete with respect to ${ }^{0} m_{E}$.

(v) For every ${ }^{0} m$-integrable function $f$,

$$
\int f d^{0} m_{E}=\int f d^{0} m
$$


In particular for every standard Lebesgue integrable function $f$

$$
\int{ }^{*} f d^{0} m_{E}=\int f d m
$$

Proof. (ii) Suppose $A \in \mathcal{L}$ then clearly ${ }^{0} m_{E}(A)<{ }^{0} m(A)$.

But, also ${ }^{0} m_{E}\left({ }^{*} I \backslash A\right)<{ }^{0} m\left({ }^{*} I \backslash A\right)$. Hence

$$
1={ }^{0} m(A)+{ }^{0} m\left({ }^{*} I \backslash A\right) \geqslant{ }^{0} m_{E}(A)+{ }^{0} m_{E}\left({ }^{*} I \backslash A\right)>0 m_{E}\left({ }^{*} I\right)=1
$$

and since everything in sight is nonnegative ${ }^{0} m(A)={ }^{0} m_{E}(A)$.

(i) If $A=B \cap E$ and $B \in \mathcal{L}$ then

$$
{ }^{0} m_{E}(A)<{ }^{0} m_{E}(B)={ }^{0} m(B) \text { and }{ }^{0} m_{E}(E \backslash A)<{ }^{0} m\left({ }^{*} I \backslash B\right) \text {. }
$$

Thus $1={ }^{0} m(B)+{ }^{0} m\left({ }^{*} I \backslash B\right)>{ }^{0} m_{E}(A)+{ }^{0} m_{E}\left({ }^{*} I \backslash A\right)>{ }^{0} m_{E}\left({ }^{*} I\right)=1$ and as above we conclude ${ }^{0} m_{E}(A)={ }^{0} m(B)$.

(iii) Suppose $A_{1}, A_{2}, \ldots$ are disjoint sets in $\mathcal{E}_{E}$ choose $B_{1}, B_{2}, \ldots$ in $\mathfrak{L}$ such that $B_{n} \cap E=A_{n} \cap E$. Let $B=\cup_{i \neq j} B_{i} \cap B_{j}$. Since the $A_{i}$ 's are disjoint $B \subseteq I \backslash E$ and since $E$ has outer measure one $m(B)=0$. Let $B_{n}^{\prime}=B_{n} \backslash B$ for each $n$. Then $A_{n} \cap E=B_{n} \cap E$ for each $n$. So ${ }^{0} m_{E}\left(A_{n}\right)$ $={ }^{0} m\left(B_{n}\right)$ and ${ }^{0} m_{E}\left(\cup A_{n}\right)={ }^{0} m\left(\cup B_{n}\right)$ and (iii) follows since ${ }^{0} m$ is $\sigma$-additive.

(iv) Suppose $A \subseteq A_{1}$ and ${ }^{0} m_{E}\left(A_{1}\right)=0$ then there is a $B_{1} \in \mathcal{L}$ such that $A_{1} \cap E=B_{1} \cap E$ and $m\left(B_{1}\right)=0$ then $B_{1} \backslash\left(A_{1} \backslash A\right) \subseteq B_{1}$ is in $\mathcal{L}$ since $\mathcal{L}$ is complete. $A \cap E=\left[B_{1} \backslash\left(A_{1} \backslash A\right)\right] \cap E$ so $A \in \mathcal{L}_{E}$.

(v) For simple ${ }^{0} m$-integrable functions (v) follows immediately from (ii). By Lebesgue's Monotone Convergence Theorem (v) follows for nonnegative $f$ and finally by the usual decomposition $f=f^{+}-f^{-}$(v) follows for ${ }^{0} m$ integrable functions. This completes the proof.

\section{REFERENCES}

1. Allen R. Bernstein and Frank Wattenberg, Nonstandard measure theory, Applications of Model Theory to Algebra, Analysis, and Probability, Holt, New York, 1969, pp. 171-185.

2. Leo Breiman, Probability, Addison-Wesley, Reading, Mass., 1968.

2A. C. Goffman, C. J. Neugebauer and T. Nishiura, Density topology and approximate continuity, Duke Math. J. 28 (1961), 497-505.

3. Ward Henson, On the nonstandard representation of measures, Trans. Amer. Math. Soc. 172 (1972), 437-446.

4. Peter Loeb, Conversion from nonstandard to standard measure spaces and applications to probability theory, Trans. Amer. Math Soc. 211 (1975), 113-122.

5. Moshe Machover and Joram Hirschfeld, Lectures on non-standard analysis, Lecture Notes in Math., vol. 94, Springer-Verlag, Berlin, 1969.

6. Rohit Parikh and Milton Parnes, Conditional probability can be defined for all pairs of sets of reals, Advances in Math. 9 (1972), 313-315.

7. Conditional probabilities and uniform sets, in Hurd and Loeb, Victoria Symposium on Nonstandard Analysis, Lecture Notes in Math., no. 369, Springer-Verlag, Berlin, 1974, pp. 180-194.

8. Abraham Robinson, Non-standard analysis, North-Holland, Amsterdam, 1974.

9. Halsey L. Royden, Real analysis, Macmillan, New York, 1968. 
10. Stanislaw Saks, Theory of the integral, Dover, New York, 1964.

11. Robert Solovay, A model of set theory in which every set of reals is Lebesgue measurable, Ann. of Math. (2) 92 (1970), 1-56.

12. Keith Stroyan and W. A. J. Luxemburg, Introduction to the theory of infinitesimals, Academic Press, New York, 1976.

13. Frank Wattenberg, Nonstandard measure theory-Hausdorff measure, Proc. Amer. Math. Soc. 65 (1977), 326-331.

Departmant of Mathematics, University of Massachusetts, AMhierst, Massachusetts 01003 